Bull. Austral. Math. Soc.

VOL. $62(2000) \quad[511-524]$

\title{
REGULAR ORDER-PRESERVING TRANSFORMATION SEMIGROUPS
}

\author{
Yupaporn Kemprasit and Thawhat ChangPhas
}

\begin{abstract}
The semigroup $O T(X)$ of all order-preserving total transformations of a finite chain $X$ is known to be regular. We extend this result to subchains of $\mathbf{Z}$; and we characterise when $O T(X)$ is regular for an interval $X$ in $\mathbf{R}$. We also consider the corresponding idea for partial transformations of arbitrary chains and posets.
\end{abstract}

\section{INTRODUCTION}

If $X$ is a set, we let $P(X)$ denote the semigroup under composition of all partial transformations of $X$ (that is, mappings $\alpha: A \rightarrow B$ where $A, B \subseteq X$ ); and we note that $P(X)$ is regular (that is, for each $\alpha \in P(X)$, there exists $\beta \in P(X)$ such that $\alpha=\alpha \beta \alpha)$. Following standard notation, we let $\operatorname{dom} \alpha$ and $\operatorname{ran} \alpha$ denote the domain and range of $\alpha \in P(X)$.

If $(X, \leqslant)$ is a poset, we say $\alpha \in P(X)$ is order-preserving if for all $x, y \in \operatorname{dom} \alpha$, $x \leqslant y$ implies $x \alpha \leqslant y \alpha$; and we let $O P(X)$ denote the semigroup under composition of all order-preserving partial transformations of $X$. Similarly, $T(X)$ denotes the semigroup of all total transformations of $X$ (that is, mappings $\alpha: X \rightarrow X$ ) and likewise it is regular. Also, if $(X, \leqslant)$ is a poset, we let $O T(X)$ denote the subsemigroup of $T(X)$ consisting of all order-preserving total transformations of $X$.

It is known $[9$, p.203, Exercise 6.1.7] that $O T(X)$ is regular if $(X, \leqslant)$ is a finite chain. In Section 2, we extend this to any chain which is order-isomorphic to a subset of $\mathbf{Z}$, the set of integers with their natural order. We also prove that if $X$ is an interval in $\mathrm{R}$, the set of real numbers, then $O T(X)$ is regular if and only if $X$ is closed and bounded. Then we answer similar questions for $O P(X)$ and some of its subsemigroups for arbitrary chains $X$. And in Section 3, we suppose $X$ is not a chain and characterise when $O P(X)$ is regular. We also list some conditions under which $O T(X)$ is (or is not) regular when $X$ is not a chain.

\section{Received 3rd May, 2000}

This paper formed part of an M.Sc. thesis written under the supervision of the first author. The second author greatly appreciates the help of his supervisor in this work; and both authors are very grateful for the assistance of Bob Sullivan, University of Western Australia, in the preparation of this paper.

Copyright Clearance Centre, Inc. Serial-fee code: 0004-9727/00 \$A2.00+0.00. 


\section{ORDER-PRESERVING TRANSFORMATIONS OF CHAINS}

If $(X, \leqslant)$ is a poset, we define the opposite partial order $\leqslant$ opp on $X$ via:

$$
x \leqslant \text { opp } y \text { if and only if } y \leqslant x .
$$

Note that if $\alpha \in P(X)$ then $\alpha$ preserves $\leqslant$ if and only if it preserves $\leqslant_{\text {opp }}$. Consequently, $O P(X, \leqslant)=O P(X, \leqslant$ opp $)$ and the regularity of $O P(X, \leqslant)$ holds equally for $O P(X, \leqslant$ opp $)$. Similar statements are true for $O T(X, \leqslant)$ and for $O I(X, \leqslant)$, the semigroup of all order-preserving partial transformations of $X$ which are one-to-one ("injective").

For the chain $\mathbf{Z}$ of integers, we let $\mathbf{Z}^{+}$denote the set of positive integers and $\mathbf{Z}^{-}$ the set of negative integers. If $X$ is a chain which is order-isomorphic to a subset of $\mathbf{Z}$ with its natural order then $X$ has one of the following forms:

(1) $\left\{x_{1}, x_{2}, \ldots, x_{n}\right\}$ where $n \in \mathbf{Z}^{+}$and $x_{1}<x_{2}<\cdots<x_{n}$,

(2) $\left\{x_{i}: i \in \mathbf{Z}^{+}\right\}$where $x_{i}<x_{j}$ if $i<j$,

(3) $\left\{x_{i}: i \in \mathbf{Z}\right\}$ where $x_{i}<x_{j}$ if $i<j$, or

(4) $\left\{x_{i}: i \in \mathbf{Z}^{-}\right\}$where $x_{i}<x_{j}$ if $i<j$.

Our first result will be needed often in what follows: its simple proof is omitted.

LEMMA 2.1. Let $X$ be a chain. If $\alpha \in O P(X)$ and $a, b \in \operatorname{ran} \alpha$ satisfy $a<b$ then $x<y$ for all $x \in a \alpha^{-1}$ and $y \in b \alpha^{-1}$.

For any $A \subseteq X$, we let $\min (A)$ and $\max (A)$ denote the minimum and the maximum elements of $A$ if they exist.

THEOREM 2.2. Let $X$ be a chain. If $X$ is order-isomorphic to a subset of $\mathbf{Z}$ then the semigroup $O T(X)$ is regular.

Proof: We regard $X$ as being one of the chains (1)-(4) listed above. Then, if $A$ is any nonempty subset of $X, \max (A)$ exists if $A$ has an upper bound in $X$, and $\min (A)$ exists if $A$ has a lower bound in $X$. It follows from this and Lemma 2.1 that, if $\alpha \in O P(X)$ and $a \in \operatorname{ran} \alpha$, then $\max \left(a \alpha^{-1}\right)$ exists if $a<b$ for some $b \in \operatorname{ran} \alpha$, and $\min \left(a \alpha^{-1}\right)$ exists if $b<a$ for some $b \in \operatorname{ran} \alpha$.

Let $\alpha \in O T(X)$. If $\alpha$ is a constant map, it is clearly regular. Therefore, suppose $\operatorname{ran} \alpha$ contains at least two elements and note that, since it is a subchain of $X$, it takes one of the forms listed in (1)-(4) above. In cases (1)-(3), we define $\beta: X \rightarrow X$ as follows:

(1) if $\operatorname{ran} \alpha=\left\{a_{1}, a_{2}, \ldots, a_{n}\right\}$, let

$$
x \beta= \begin{cases}\max \left(a_{1} \alpha^{-1}\right) & \text { if } x \leqslant a_{1}, \\ \min \left(a_{i+1} \alpha^{-1}\right) & \text { if } a_{i}<x \leqslant a_{i+1} \text { and } i \neq n, \\ \min \left(a_{n} \alpha^{-1}\right) & \text { if } x \geqslant a_{n} .\end{cases}
$$


(2) if $\operatorname{ran} \alpha=\left\{a_{i}: i \in \mathbf{Z}^{+}\right\}$, let

$$
x \beta= \begin{cases}\max \left(a_{1} \alpha^{-1}\right) & \text { if } x \leqslant a_{1}, \\ \min \left(a_{i+1} \alpha^{-1}\right) & \text { if } a_{i}<x \leqslant a_{i+1} \text { for some } i \in \mathbf{Z}^{+} .\end{cases}
$$

(3) if $\operatorname{ran} \alpha=\left\{a_{i}: i \in \mathbf{Z}\right\}$, let

$$
x \beta=\max \left(a_{i+1} \alpha^{-1}\right) \text { if } a_{i}<x \leqslant a_{i+1} \text { for some } i \in \mathbf{Z} .
$$

Now, if $x \in X$ then $x \alpha=a_{k}$ for some $k \in \mathbf{Z}$. By definitions (1)-(3), $a_{k} \beta$ equals $\max \left(a_{k} \alpha^{-1}\right)$ or $\min \left(a_{k} \alpha^{-1}\right)$ : in each case, this means $\left(a_{k} \beta\right) \alpha=a_{k}$, and hence $x(\alpha \beta \alpha)=x \alpha$ for all $x \in X$; that is, $\alpha=\alpha \beta \alpha$.

To show $\beta$ is order-preserving, suppose $x<y$ in $X$. In cases (1) and (2), if $y \leqslant a_{1}$ then $x \beta=y \beta$, and the same conclusion holds in case (1) if $x \geqslant a_{n}$. On the other hand, in each of (1)-(3), if $a_{k}<x<y \leqslant a_{k+1}$ for some $k$ then $x \beta=y \beta$, by the definition of $\beta$. Suppose instead that

$$
a_{k}<x \leqslant a_{k+1} \leqslant a_{\ell}<y \leqslant a_{\ell+1}
$$

for some $k, \ell$. Then $a_{k+1}<a_{\ell+1}$ and Lemma 2.1 imply that $u<v$ for all $u \in a_{k+1} \alpha^{-1}$ and $v \in a_{\ell+1} \alpha^{-1}$. But, by the definition of $\beta, x \beta \in a_{k+1} \alpha^{-1}$ and $y \beta \in a_{\ell+1} \alpha^{-1}$, hence $x \beta<y \beta$ as required. The remaining possibilities: $x \leqslant a_{1}<a_{n} \leqslant y$ in case (1), and $x \leqslant a_{1} \leqslant a_{\ell}<y \leqslant a_{\ell+1}$ in cases (1) and (2), lead to the same conclusion. Therefore, $\beta \in O T(X)$ is regular in cases (1)-(3).

For case (4), we recall from the start of this section that $O T\left(\mathbf{Z}^{+}, \leqslant\right)=O T\left(\mathbf{Z}^{+}, \leqslant\right.$opp $)$, and clearly $\left(\mathbf{Z}^{-}, \leqslant\right)$is order-isomorphic to $\left(\mathbf{Z}^{+}, \leqslant\right.$opp $)$. Hence, from our conclusion in case (3), if $X$ is order-isomorphic to $\mathbf{Z}^{-}$then $O T(X)$ is regular.

We now show that $O T(\mathbf{R})$ is not regular when $\mathbf{R}$ is the set of real numbers with their natural order. In fact, the following sequence of Lemmas will eventually characterise when $O T(X)$ is regular for an interval $X$ in $\mathbf{R}$.

LEMma 2.3. The semigroup $O T(\mathbf{R})$ is not regular.

Proof: Fix $r \in(1, \infty)$ and let $\alpha \in O T(\mathbf{R})$ be the map: $x \alpha=r^{x}$ for all $x \in \mathbf{R}$. Then $\operatorname{ran} \alpha=\mathbf{R}^{+}$and $\alpha$ is one-to-one. Suppose $\alpha=\alpha \beta \alpha$ for some $\beta \in O T(\mathbf{R})$. Then, since $\alpha$ is one-to-one, $x=x \alpha \beta$ for each $x \in \mathbf{R}$. Thus, $\mathbf{R}^{+} \beta=\mathbf{R}$. Hence, since $0 \beta \in \mathbf{R}$, there exists $d \in \mathbf{R}^{+}$such that $0 \beta=d \beta$. Choose $c \in(0, d)$. Then

$$
0 \beta \leqslant c \beta \leqslant d \beta=0 \beta,
$$

and we deduce that $c \beta=d \beta$. Since $c, d \in \mathbf{R}^{+}=\operatorname{ran} \alpha$, we can choose $x, y \in \mathbf{R}$ with $x \alpha=c$ and $y \alpha=d$. Then

$$
c=x \alpha=x \alpha \beta \alpha=c \beta \alpha=d \beta \alpha=y \alpha \beta \alpha=y \alpha=d
$$


which contradicts $c<d$. Hence, $\alpha$ is not a regular element of $O T(\mathbf{R})$, and the result follows.

LEMMA 2.4. The semigroup $\operatorname{OT}((a, \infty))$ is not regular for any $a \in \mathbf{R}$.

Proof: Fix $r \in[1, \infty)$ and let $\alpha \in O T((a, \infty))$ be the map: $x \alpha=x+r$ for all $x \in(a, \infty)$. Then $\operatorname{ran} \alpha=(a+r, \infty)$ and $\alpha$ is one-to-one. Suppose $\alpha=\alpha \beta \alpha$ for some $\beta \in O T((a, \infty))$. Then, since $\alpha$ is one-to-one, $x=x \alpha \beta$ for each $x \in(a, \infty)$. Thus, $(a+r, \infty) \beta=(a, \infty)$. Hence, since $(a+r) \beta \in(a, \infty)$, there exists $d \in(a+r, \infty)$ such that $(a+r) \beta=d \beta$. Choose $c \in(a+r, d)$. Then

$$
(a+r) \beta \leqslant c \beta \leqslant d \beta=(a+r) \beta
$$

and we deduce that $c \beta=d \beta$. Since $c, d \in(a+r, \infty)=\operatorname{ran} \alpha$, we can choose $x, y \in$ $(a, \infty)$ with $x \alpha=c$ and $y \alpha=d$. Then

$$
c=x \alpha=x \alpha \beta \alpha=c \beta \alpha=d \beta \alpha=y \alpha \beta \alpha=y \alpha=d
$$

which contradicts $c<d$. Hence, $\alpha$ is not a regular element of $O T((a, \infty))$, and the result follows.

LEMma 2.5. The semigroup $O T([a, \infty))$ is not regular for any $a \in \mathbf{R}$.

Proof: Fix $r \in[1, \infty)$ and let $\alpha$ be the map:

$$
x \alpha=a+\frac{x-a}{x-a+r} \text { for all } x \in[a, \infty)
$$

Then $\operatorname{ran} \alpha=[a, a+1)$. Moreover, since the derivative of $\alpha$ is strictly positive on $(a, \infty)$, we know $\alpha$ is increasing and hence it is one-to-one. Therefore, $\alpha \in O T([a, \infty))$. Suppose $\alpha=\alpha \beta \alpha$ for some $\beta \in O T([a, \infty))$. Then, since $\alpha$ is one-to-one, $x=x \alpha \beta$ for each $x \in[a, \infty)$, and so $[a, a+1) \beta=[a, \infty)$. Hence, since $(a+1) \beta \in[a, \infty)$, we know $(a+1) \beta=d \beta$ for some $d \in[a, a+1)$. Choose $c \in(d, a+1)$. Then

$$
d \beta \leqslant c \beta \leqslant(a+1) \beta=d \beta,
$$

and we deduce that $c \beta=d \beta$. Since $c, d \in[a, a+1)=\operatorname{ran} \alpha$, we can choose $x, y \in$ $[a, \infty)$ with $x \alpha=c$ and $y \alpha=d$. The result then follows as in the proofs of the last Lemmas.

LEMMA 2.6. The semigroup $\operatorname{OT}((a, b))$ is not regular for any $a, b \in \mathbf{R}$ with $a<b$.

PRoOF: Fix $r \in(0, b-a)$ and let $\alpha$ be the map:

$$
x \alpha=\left(1-\frac{r}{b-a}\right) x+\frac{r b}{b-a} \text { for all } x \in(a, b) .
$$


That is, the graph of $\alpha$ is a line segment with positive slope, and clearly $\operatorname{ran} \alpha=$ $(a+r, b)$. Therefore, $\alpha \in O T((a, b))$. Suppose $\alpha=\alpha \beta \alpha$ for some $\beta \in O T((a, b))$. Then, since $\alpha$ is one-to-one, $x=x \alpha \beta$ for each $x \in(a, b)$, and so $(a+r, b) \beta=(a, b)$. Hence, since $(a+r) \beta \in(a, b)$, we know $(a+r) \beta=d \beta$ for some $d \in(a+r, b)$. Choose $c \in(a+r, d)$. Then

$$
d \beta=(a+r) \beta \leqslant c \beta \leqslant d \beta,
$$

and we deduce that $c \beta=d \beta$. Since $c, d \in(a+r, b)=\operatorname{ran} \alpha$, we can choose $x, y \in(a, b)$ with $x \alpha=c$ and $y \alpha=d$, and the result follows as before.

LEMma 2.7. The semigroup $O T([a, b))$ is not regular for any $a, b \in \mathbf{R}$ with $a<b$.

Proof: Fix $r \in(0, b-a)$ and let $\alpha$ be the map:

$$
x \alpha=\frac{r x}{b-a}+a-\frac{r a}{b-a} \text { for all } x \in[a, b) .
$$

That is, the graph of $\alpha$ is a line segment with positive slope, and clearly $\operatorname{ran} \alpha=$ $[a, a+r)$. Therefore, $\alpha \in O T([a, b))$. Suppose $\alpha=\alpha \beta \alpha$ for some $\beta \in O T([a, b))$. Then, since $\alpha$ is one-to-one, $x=x \alpha \beta$ for each $x \in[a, b)$, and so $[a, a+r) \beta=[a, b]$. Hence, since $(a+r) \beta \in[a, b)$, we know $(a+r) \beta=d \beta$ for some $d \in[a, a+r)$. Choose $c \in(d, a+r)$. Then

$$
d \beta \leqslant c \beta \leqslant(a+r) \beta=d \beta,
$$

and we deduce that $c \beta=d \beta$. Since $c, d \in[a, a+r)=\operatorname{ran} \alpha$, we can choose $x, y \in[a, b)$ with $x \alpha=c$ and $y \alpha=d$, and the result follows as before.

With the notation at the start of this section, if $\leqslant$ is the natural order on $\mathbf{R}$ then

(1) $((-\infty, a), \leqslant)$ is order-isomorphic to $((-a, \infty), \leqslant$ opp $)$ for each $a \in \mathbf{R}$,

(2) $((-\infty, a], \leqslant)$ is order-isomorphic to $([-a, \infty), \leqslant$ opp $)$ for each $a \in \mathbf{R}$, and

(3) $((a, b], \leqslant)$ is order-isomorphic to $([-b,-a), \leqslant$ opp $)$ for each $a, b \in \mathbf{R}$.

Consequently, Lemmas $2.4,2.5$ and 2.7 show that the semigroups $O T((-\infty, a))$, $O T((-\infty, a])$ and $O T((a, b])$ are not regular for any $a, b \in \mathbf{R}$. This covers all nonempty intervals of $\mathbf{R}$ except one: namely, $[a, b]$ with $a<b$ and we shall prove that the semigroup $O T([a, b])$ is regular. But, to do this, we need one more Lemma.

Lemma 2.8. Let $\alpha \in O T([a, b])$ where $a, b \in \mathbf{R}$ and $a<b$, and suppose $x \in$ $(a \alpha, b \alpha)$. If $A_{x}=[a, x] \alpha^{-1}$ and $B_{x}=(x, b] \alpha^{-1}$ then $\left\{A_{x}, B_{x}\right\}$ is a partition of $[a, b]$ such that $c<d$ for all $c \in A_{x}$ and $d \in B_{x}$.

Proof: Since $x \in(a \alpha, b \alpha)$, we know $a \leqslant a \alpha<x$ and $x<b \alpha \leqslant b$, so $a \epsilon$ $[a, x] \alpha^{-1}=A_{x}$ and $b \in(x, b] \alpha^{-1}=B_{x}$. The result then follows from Lemma 2.1 and the fact that $[a, b]=[a, x] \cup(x, b]$. 
For the next result, we recall: if $I$ is an interval in $\mathbf{R}$ and if $\{A, B\}$ is a partition of $I$ such that $x<y$ for all $x \in A$ and $y \in B$ then either $\max (A)$ or $\min (B)$ exists (but not both).

Lemma 2.9. The semigroup $O T([a, b])$ is regular for any $a, b \in \mathbf{R}$ with $a<b$.

Proof: Let $\alpha \in O T([a, b])$ and note that $a \alpha \leqslant b \alpha$ and $\operatorname{ran} \alpha \subseteq[a \alpha, b \alpha]$. Define $d_{x}$ for each $x \in[a, b]$ as follows:

$$
d_{x} \begin{cases}=a & \text { if } x \in[a, a \alpha) \\ =b & \text { if } x \in(b \alpha, b] \\ \in x \alpha^{-1} & \text { if } x \in \operatorname{ran} \alpha .\end{cases}
$$

To complete the definition, suppose $x \in(a \alpha, b \alpha) \backslash \operatorname{ran} \alpha$ and put $A_{x}=[a, x] \alpha^{-1}$ and $B_{x}=(x, b] \alpha^{-1}$. By Lemma $2.8,\left\{A_{x}, B_{x}\right\}$ is a partition of $[a, b]$ with a special property; and, by a remark above, either $\max \left(A_{x}\right)$ or $\min \left(B_{x}\right)$ exists (but not both). Hence, we can define:

$$
d_{x}= \begin{cases}\max \left(A_{x}\right) & \text { if } x \in(a \alpha, b \alpha) \backslash \operatorname{ran} \alpha \text { and } \max \left(A_{x}\right) \text { exists, } \\ \min \left(B_{x}\right) & \text { if } x \in(a \alpha, b \alpha) \backslash \operatorname{ran} \alpha \text { and } \min \left(B_{x}\right) \text { exists. }\end{cases}
$$

Finally, we let $\beta:[a, b] \rightarrow[a, b], x \rightarrow d_{x}$. If $x \in[a, b]$ then $x \alpha \in \operatorname{ran} \alpha$, so the definition of $d_{x}$ implies that $d_{x \alpha} \alpha=x \alpha$. Hence,

$$
x \alpha \beta \alpha=((x \alpha) \beta) \alpha=d_{x \alpha} \alpha=x \alpha
$$

which shows that $\alpha=\alpha \beta \alpha$ on $[a, b]$.

To show that $\beta$ is order-preserving, let $x, y \in[a, b]$ and $x<y$. Then $x \in[a, y]$ and $y \in(x, b]$, and we consider six cases.

CASE 1. $x<a \alpha$. Then $x \beta=d_{x}=a$, so $x \beta \leqslant y \beta$.

CASE 2. $y>b \alpha$. Then $y \beta=d_{y}=b$, so $x \beta \leqslant y \beta$.

CASE 3. $x, y \in \operatorname{ran} \alpha$. By Lemma 2.1, $u<v$ for all $u \in x \alpha^{-1}$ and $v \in y \alpha^{-1}$. In particular, by definition, $x \beta=d_{x}<d_{y}=y \beta$.

CASE 4. $x \in \operatorname{ran} \alpha$ and $y \in(a \alpha, b \alpha) \backslash \operatorname{ran} \alpha$. Then $d_{x} \in x \alpha^{-1} \subseteq[a, y] \alpha^{-1}=A_{y}$. Hence, if $\max \left(A_{y}\right)$ exists then

$$
x \beta=d_{x} \leqslant \max \left(A_{y}\right)=d_{y}=y \beta .
$$

On the other hand, if $\min \left(B_{y}\right)$ exists then, by Lemma 2.8 , we have:

$$
x \beta=d_{x}<d_{y}=\min \left(B_{y}\right)=y \beta .
$$

CASE 5. $x \in(a \alpha, b \alpha) \backslash \operatorname{ran} \alpha$ and $y \in \operatorname{ran} \alpha$. An argument similar to that in case (4) shows $x \beta \leqslant y \beta$ in this case also. 
CASE 6. $x, y \in(a \alpha, b \alpha) \backslash \operatorname{ran} \alpha$. If $[x, y] \cap \operatorname{ran} \alpha=\emptyset$ then

$$
A_{x}=[a, x] \alpha^{-1}=[a, y] \alpha^{-1}=A_{y} \text { and } B_{x}=(x, b] \alpha^{-1}=(y, b] \alpha^{-1}=B_{y}
$$

and hence, by definition, $x \beta=d_{x}=d_{y}=y \beta$. However, if $[x, y] \cap \operatorname{ran} \alpha \neq \emptyset$ then there exists $c \in \operatorname{ran} \alpha$ such that $x<c<y$. In this event, we can choose $p \in[a, b]$ with $p \alpha=c$. Then

$$
p \in[a, y] \alpha^{-1} \cap(x, b] \alpha^{-1}=A_{y} \cap B_{x}
$$

and so, using a standard property of $\mathbf{R}$, we have:

$$
\sup \left(A_{x}\right) \leqslant \inf \left(B_{x}\right) \leqslant p \leqslant \sup \left(A_{y}\right) \leqslant \inf \left(B_{y}\right) .
$$

Note that $\sup \left(A_{x}\right)$ equals $\max \left(A_{x}\right)$ if this maximum exists, and it equals $\min \left(B_{x}\right)$ if this minimum exists; and a similar comment can be made for $\inf \left(B_{y}\right)$. Hence, we conclude from the above that $d_{x} \leqslant d_{y}$ and so $x \beta \leqslant y \beta$.

The combination of Lemmas 2.3-2.9, and the remarks between them, give us the following result.

THEOREM 2.10. For any interval $X$ of $\mathrm{R}$, the semigroup $O T(X)$ is regular if and only if $X$ is closed and bounded.

In passing, we note that in [11] Howie showed that $O T(X)$ is also idempotentgenerated if $X$ is a finite chain, and in [8] the authors extended this to $O P(X)$, while in [7] Garba investigated the same idea for various subsemigroups of $O P(X)$ : see [12] for a brief survey of this and related work; and see [10] for an alternative approach to the same idea for $O T(X)$ and its subsemigroup consisting of all decreasing transformations (that is, $x \alpha \leqslant x$ for all $x \in X$ ). For an arbitrary chain $X$, the elements of $O T(X)$ which are products of idempotents were described in [14]; and the corresponding notion for products of "nilpotents" in $O P(X)$ and $O I(X)$ has been examined in [6] and [5] for finite chains.

In [3] the authors considered the semigroup $O P^{\prime}(X)$ consisting of all orderpreserving transformations $\alpha$ whose domains are final segments in a chain $X$ (that is, $x \in \operatorname{dom} \alpha$ and $x \leqslant y$ imply $y \in \operatorname{dom} \alpha)$; and they observed that this semigroup need not be regular. By contrast with this fact and the above results for $O T(X)$, we prove the following Theorem.

THEOREM 2.11. If $X$ is a chain then the semigroup $O P(X)$ is regular.

Proof: Let $\alpha \in O P(X)$ and, for each $a \in \operatorname{ran} \alpha$, choose $d_{a} \in a \alpha^{-1}$. Define a partial transformation $\beta$ via: $\operatorname{dom} \beta=\operatorname{ran} \alpha$ and $a \beta=d_{a}$ for each $a \in \operatorname{ran} \alpha$. Then $x(\alpha \beta \alpha)=x \alpha$ for all $x \in \operatorname{dom} \alpha$ (since $d_{a} \alpha=a$ for all $a \in \operatorname{ran} \alpha$ ) and in fact 
$\operatorname{dom}(\alpha \beta \alpha)=\operatorname{dom} \alpha$. Hence, $\alpha=\alpha \beta \alpha$. Also, if $a<b$ in $\operatorname{dom} \beta=\operatorname{ran} \alpha$ then $d_{a}<d_{b}$ by Lemma 2.1 , so $\beta$ is order-preserving, and the result follows.

As usual, if $X$ is a set and $\alpha \in P(X)$, we define the shift of $\alpha$ to be $s(\alpha)=|S(\alpha)|$ where

$$
S(\alpha)=\{x \in \operatorname{dom} \alpha: x \alpha \neq x\}
$$

and we write

$$
P\left(X, \aleph_{0}\right)=\left\{\alpha \in P(X): s(\alpha)<\aleph_{0}\right\} .
$$

It is well-known that $P\left(X, \aleph_{0}\right)$ is a semigroup: it is sometimes called the semigroup of almost identical partial transformations of $X$ [15]. If $X$ is a poset, we let $O P\left(X, \aleph_{0}\right)$ denote the semigroup of all order-preserving partial transformations of $X$ with finite shift, and $O I\left(X, \aleph_{0}\right)$ will denote the semigroup of all one-to-one transformations in $O P\left(X, \aleph_{0}\right)$.

If $X$ is a chain and $\alpha \in O P\left(X, \aleph_{0}\right)$, we can define a map $\beta \in O P(X)$ as in the proof of Theorem 2.11 so that $\alpha=\alpha \beta \alpha$. In fact, since $a \alpha^{-1}=\{a\}$ for all $a \in \operatorname{ran} \alpha \backslash S(\alpha) \alpha$, we have $a \beta=a$ for all $a \in \operatorname{ran} \alpha \backslash S(\alpha) \alpha$ and so $S(\beta) \subseteq S(\alpha) \alpha$ (since $\operatorname{dom} \beta=\operatorname{ran} \alpha$ ). Hence, $\beta \in O P\left(X, \aleph_{0}\right)$ and we have proved that $O P\left(X, \aleph_{0}\right)$ is regular.

In passing, we note that Lavers [13] considered certain subsemigroups of $O P\left(Z^{+}, \aleph_{0}\right)$ in a different context, with the aim of giving presentations for them and describing their principal left (right) ideals.

In [4, Proposition 1.4], Fernandes noted that $O I(X)$ is regular if $X$ is a finite chain. In fact, following the proof of Theorem 2.11, it is clear that if $X$ is any chain and $\alpha \in O I(X)$ then there exists $\beta \in O I(X)$ with $\alpha=\alpha \beta \alpha$. So, $O I(X)$ is regular for any chain $X$. Indeed, since the idempotents of $O I(X)$ are simply those transformations which fix a subchain of $X$ pointwise, and hence they commute, we deduce that $O I(X)$ is an inverse semigroup. The significance of $O I(X)$ is illustrated by a result in [2]: namely, any set $X$ with $|X| \neq 3$ can be ordered so that $O I(X)$ forms a transversal of the set of $\mathcal{H}$-classes in $I(X)$.

Finally, we consider the semigroup:

$$
O T\left(X, \aleph_{0}\right)=\left\{\alpha \in O T(X): s(\alpha)<\aleph_{0}\right\}
$$

and aim to show it is regular if $X$ is a chain. However, for this we need another three Lemmas.

Lemma 2.12. Let $X$ be a poset, $\alpha \in O P(X)$ and $a \in \operatorname{dom} \alpha$. Then $\{x \in \operatorname{dom} \alpha: a \alpha<x<a\} \subseteq S(\alpha)$ and $\{x \in \operatorname{dom} \alpha: a<x<a \alpha\} \subseteq S(\alpha)$.

PRoof: If $x \in \operatorname{dom} \alpha$ and $a \alpha<x<a$ then $x \alpha \leqslant a \alpha$. Thus, if $x \alpha=x$, we have $x \leqslant a \alpha$, a contradiction; so, $x \in S(\alpha)$ as required. The other containment follows similarly. 
Lemma 2.13. Let $X$ be a poset, $\alpha \in O P(X)$ and $A \subseteq \operatorname{ran} \alpha$. If $\max (A)$ and $\max \left(A \alpha^{-1}\right)$ exist then $\max (A)=\left[\max \left(A \alpha^{-1}\right)\right] \alpha$.

Proof: Since $\max (A) \in A \subseteq \operatorname{ran} \alpha$, there exists $x \in \operatorname{dom} \alpha$ such that $\max (A)=$ $x \alpha$. Then $x \in A \alpha^{-1}$, so $x \leqslant \max \left(A \alpha^{-1}\right)$ and, since $\alpha$ is order-preserving, we deduce that $\max (A) \leqslant\left[\max \left(A \alpha^{-1}\right)\right] \alpha$. Since $\max \left(A \alpha^{-1}\right) \in A \alpha^{-1}$ and $A \subseteq \operatorname{ran} \alpha$, we know $\left[\max \left(A \alpha^{-1}\right)\right] \alpha \in A$ and this implies $\left[\max \left(A \alpha^{-1}\right)\right] \alpha \leqslant \max (A)$. Hence, equality holds as required.

Lemma 2.14. Let $X$ be a poset, $\alpha \in O P(X)$ and $A, B \subseteq \operatorname{ran} \alpha$, and suppose $\max A, \max B, \max \left(A \alpha^{-1}\right)$ and $\max \left(B \alpha^{-1}\right)$ exist.

(1) If $\max A=\max B$ then $\max \left(A \alpha^{-1}\right)=\max \left(B \alpha^{-1}\right)$.

(2) If $X$ is a chain and $\max A<\max B$ then $\max \left(A \alpha^{-1}\right)<\max \left(B \alpha^{-1}\right)$.

Proof: By Lemma 2.13, $\left[\max \left(A \alpha^{-1}\right)\right] \alpha=\max A$. Therefore, if $\max A=$ $\max B$, we have $\left[\max \left(A \alpha^{-1}\right)\right] \alpha \in B$ and hence $\max \left(A \alpha^{-1}\right) \in B \alpha^{-1}$. Consequently, $\max \left(A \alpha^{-1}\right) \leqslant \max \left(B \alpha^{-1}\right)$, and a dual argument establishes equality in (1). On the other hand, if $\max A<\max B$ then Lemma 2.13 implies $\left[\max \left(A \alpha^{-1}\right)\right] \alpha<$ $\left[\max \left(B \alpha^{-1}\right)\right] \alpha$. Therefore, if $X$ is a chain, we must have $\max \left(A \alpha^{-1}\right)<\max \left(B \alpha^{-1}\right)$ (since $\alpha$ is order-preserving).

We can now prove the following result.

THEOREM 2.15. If $X$ is a chain then the semigroup $O T\left(X, \aleph_{0}\right)$ is regular.

Proof: Let $\alpha \in O T\left(X, \aleph_{0}\right)$ and note that $\operatorname{dom} \alpha=X$. For each $x \in X$, we define $d_{x} \in X$ as follows.

CASE I. $x \in \operatorname{ran} \alpha$. Since $x \alpha^{-1}$ is nonempty and finite for each $x \in \operatorname{ran} \alpha$, and $X$ is a chain, we know $\max \left(x \alpha^{-1}\right)$ always exists in this case. So, we put

$$
d_{x}=\max \left(x \alpha^{-1}\right) \quad \text { if } x \in \operatorname{ran} \alpha .
$$

CASE II. $x \notin \operatorname{ran} \alpha$. In this case, $x \in S(\alpha)$ and this implies $x \alpha<x$ or $x<x \alpha$ since $X$ is a chain. Then, from Lemma 2.12, we deduce that $\{z \in X: x \alpha<z<x\}$ and $\{z \in X: x<z<x \alpha\}$ are finite sets. In turn this implies $\{y \in \operatorname{ran} \alpha: x \alpha \leqslant y<x\}=C$ say, and $\{y \in \operatorname{ran} \alpha: x<y \leqslant x \alpha\}=D$ say, are finite sets. Therefore, since $y \alpha^{-1}$ is finite for each $y \in \operatorname{ran} \alpha$, the inverse images of $C$ and $D$ are also finite. Hence, if $x \alpha<x$ then $C \neq \emptyset$ and $\max \left(C \alpha^{-1}\right)$ exists; and if $x<x \alpha$ then $D \neq \emptyset$ and $\min \left(D \alpha^{-1}\right)$ exists. So, in this case, we put

$$
d_{x}= \begin{cases}\max \left(\{y \in \operatorname{ran} \alpha: x \alpha \leqslant y<x\} \alpha^{-1}\right) & \text { if } x \alpha<x, \\ \min \left(\{y \in \operatorname{ran} \alpha: x<y \leqslant x \alpha\} \alpha^{-1}\right) & \text { if } x<x \alpha .\end{cases}
$$


Now let $\beta: X \rightarrow X, x \rightarrow d_{x}$ and note that $\alpha=\alpha \beta \alpha$ as in the proof of Theorem 2.11. Also note that

$$
\left\{x \in \operatorname{ran} \alpha: \max \left(x \alpha^{-1}\right) \neq x\right\} \subseteq\left\{x \in \operatorname{ran} \alpha: x \alpha^{-1} \neq\{x\}\right\}
$$

and the second set in this display is finite since $S(\alpha)$ is finite. By definition of $\beta$, this means $\{x \in \operatorname{ran} \alpha: x \beta \neq x\}$ is finite. But we have:

$$
S(\beta) \subseteq(X \backslash \operatorname{ran} \alpha) \cup\{x \in \operatorname{ran} \alpha: x \beta \neq x\}
$$

where both sets in this union are finite. Hence, $\beta \in T\left(X, \aleph_{0}\right)$.

To show $\beta$ is order-preserving, suppose $a<b$ in $X$ and consider four cases.

CASE 1. $a, b \in \operatorname{ran} \alpha$. Then, by definition, $d_{a} \in a \alpha^{-1}$ and $d_{b} \in b \alpha^{-1}$, so $d_{a}<d_{b}$ by Lemma 2.1 .

CASE 2. $a \in \operatorname{ran} \alpha$ and $b \notin \operatorname{ran} \alpha$. Then $b \alpha \neq b$, so $b \alpha<b$ or $b<b \alpha$ since $X$ is a chain, and we consider two possibilities.

(i) Suppose $b \alpha<b$. If $a \in\{y \in \operatorname{ran} \alpha: b \alpha \leqslant y<b\}=B$ say, then $\max \left(a \alpha^{-1}\right) \leqslant \max \left(B \alpha^{-1}\right)$ which implies $d_{a} \leqslant d_{b}$. On the other hand, if $a \notin B$ then, since $a \in \operatorname{ran} \alpha$ and $a<b$ by supposition, we must have $a<b \alpha$ and so $a<y$ for all $y \in B$. Hence, Lemma 2.1 implies $u<v$ for all $u \in a \alpha^{-1}$ and $v \in B \alpha^{-1}$, and so $\max \left(a \alpha^{-1}\right)<\max \left(B \alpha^{-1}\right)$ : that is, $d_{a}<d_{b}$.

(ii) Suppose $b<b \alpha$. Then $a<b<b \alpha$, so $a<y$ for all $y \in \operatorname{ran} \alpha$ such that $b<y \leqslant b \alpha$. Hence, Lemma 2.1 implies

$$
\max \left(a \alpha^{-1}\right)<\min \left(\{y \in \operatorname{ran} \alpha: b<y \leqslant b \alpha\} \alpha^{-1}\right)
$$

and we have shown $d_{a}<d_{b}$.

CASE 3. $a \notin \operatorname{ran} \alpha$ and $b \in \operatorname{ran} \alpha$. In this case, $a \alpha<a$ or $a<a \alpha$. Since $a<b$, the first possibility leads to

$$
\max \left(\{y \in \operatorname{ran} \alpha: a \alpha \leqslant y<a\} \alpha^{-1}\right)<\max \left(b \alpha^{-1}\right)
$$

and so $d_{a}<d_{b}$. If the second possibility occurs then $a<b \leqslant a \alpha$ or $a \alpha<b$, and an argument similar to that in the first paragraph of Case 2 leads to

$$
\min \left(\{y \in \operatorname{ran} \alpha: a<y \leqslant a \alpha\} \alpha^{-1}\right) \leqslant \max \left(b \alpha^{-1}\right)
$$

and it follows that $d_{a} \leqslant d_{b}$. 
CASE 4. $a \notin \operatorname{ran} \alpha$ and $b \notin \operatorname{ran} \alpha$. Then $a \alpha<a$ or $a<a \alpha$, and similarly for $b$. So, we put

$$
A=\{y \in \operatorname{ran} \alpha: a \alpha \leqslant y<a\} \text { and } B=\{y \in \operatorname{ran} \alpha: b \alpha \leqslant y<b\}
$$

and consider four possibilities.

(i) Suppose $a \alpha<a$ and $b \alpha<b$. Since $X$ is a chain, we have

$a \alpha \leqslant y<b$ if and only if $b \alpha \leqslant y<b$ or $a \alpha \leqslant y<b \alpha$.

Moreover, $\max B$ exists (as in the definition of $d_{x}$ in Case II) and this is greater than all $y \in \operatorname{ran} \alpha$ such that $a \alpha \leqslant y<b \alpha$. Hence, $\max \{y \in$ $\operatorname{ran} \alpha: a \alpha \leqslant y<b\}$ exists and it equals $\max B$. Then $a<b$ implies

$\max A \leqslant \max \{y \in \operatorname{ran} \alpha: a \alpha \leqslant y<b\}=\max B$

and so Lemma 2.14 implies $\max \left(A \alpha^{-1}\right) \leqslant \max \left(B \alpha^{-1}\right)$, so $d_{a} \leqslant d_{b}$ as required.

(ii) Suppose $a \alpha<a$ and $b<b \alpha$. Then $a \alpha<a<b<b \alpha$ and so $u<u^{\prime}$ for all $u \in A$ and $u^{\prime} \in\{y \in \operatorname{ran} \alpha: b<y \leqslant b \alpha\}$. Hence, $v<v^{\prime}$ for all $v \in A \alpha^{-1}$ and $v^{\prime} \in\{y \in \operatorname{ran} \alpha: b<y \leqslant b \alpha\} \alpha^{-1}$, and it follows that

$$
\max \left(A \alpha^{-1}\right)<\min \left(\{y \in \operatorname{ran} \alpha: b<y \leqslant b \alpha\} \alpha^{-1}\right) .
$$

Thus, $d_{a}<d_{b}$ for this possibility.

(iii) Suppose $a<a \alpha$ and $b \alpha<b$. Then $a<a \alpha \leqslant b \alpha<b$ and so $u \leqslant u^{\prime}$ for all $u \in\{y \in \operatorname{ran} \alpha: a<y \leqslant a \alpha\}$ and $u^{\prime} \in B$. Hence,

$$
\max \{y \in \operatorname{ran} \alpha: a<y \leqslant a \alpha\} \leqslant \max B
$$

and, using Lemma 2.14 , we obtain

$$
\min \left(\{y \in \operatorname{ran} \alpha: a<y \leqslant a \alpha\} \alpha^{-1}\right) \leqslant \max \left(B \alpha^{-1}\right) .
$$

Thus, $d_{a} \leqslant d_{b}$ for this possibility.

(iv) Suppose $a<a \alpha$ and $b<b \alpha$. An argument dual to that in (i), which uses the dual of Lemma 2.14 , shows that $d_{a} \leqslant d_{b}$ for this possibility.

Hence, $a<b$ implies $a \beta \leqslant b \beta$ in all possible cases, and the Theorem is completely proved. 


\section{ORDER-PRESERVING TRANSFORMATIONS OF NON-CHAINS}

Very little (if any) research appears to have been done on semigroups of orderpreserving transformations of arbitrary posets. In this section, we suppose $X$ is not a chain and determine when $O P(X)$ is regular, and then we state conditions under which $O T(X)$ is regular. We need two preliminary results, the first of which we are unable to find in the literature. If $X$ is a poset, we say $a \in X$ is isolated if for every $x \in X, x \leqslant a$ or $x \geqslant a$ implies $x=a$, and $X$ is isolated if all its elements are isolated.

LEMma 3.1. Suppose $X$ is a poset which is not a chain. If $X$ is not isolated then it contains a subposet with one of the following forms:

$$
\begin{aligned}
& \Pi_{1}=\{a, b, c:\{a, c\} \text { and }\{b, c\} \text { are isolated, and } a<b\} \\
& \Pi_{2}=\{a, b, c:\{b, c\} \text { is isolated, and } a<b, a<c\}, \\
& \Pi_{3}=\{a, b, c:\{b, c\} \text { is isolated, and } b<a, c<a\} .
\end{aligned}
$$

Proof: Since $X$ is not isolated, it contains a non-isolated element $a$, say. Then there exists $b \in X$ with $a<b$ or $b<a$ and, without loss of generality, we assume $a<b$. By Zorn's Lemma, there is a maximal chain $M$ in $X$ containing $a$ and $b$; and, since $X$ is not a chain, we can choose $c \in X \backslash M$. If $c$ is not comparable with any element of $M$, we obtain $\Pi_{1}$. On the other hand, suppose $d<c$ for some $d \in M$ and note that, in this case, if $y \in M$ and $y<d$ then $y<c$. Therefore, if for every $x \in M, d<x$ implies $c<x$ or $x<c$, we deduce that $M \cup\{c\}$ is a chain, contradicting the maximality of $M$. Hence, there exists $e \in M$ such that $d<e$ and $c \nless e$ and $e \nless c$, and we obtain $\Pi_{2}$. Finally, if $c<d$ for some $d \in M$, the dual of the above argument gives us $\Pi_{3}$.

LEMMA 3.2. Suppose $X$ is a poset which contains a subposet of the form $\Pi_{1}, \Pi_{2}$ or $\Pi_{3}$. If $S$ denotes one of the semigroups $O P(X), O I(X), O P\left(X, \aleph_{0}\right)$ or $O I\left(X, \aleph_{0}\right)$ then $S$ is not regular.

Proof: We consider three cases.

CASE 1. $X$ contains $\Pi_{1}$. Let $\alpha \in P(X)$ satisfy: $\operatorname{dom} \alpha=\{a, c\}$ and $a \alpha=b, c \alpha=a$. Then, $\alpha \in S$ since $\{a, c\}$ is isolated. Suppose $\alpha=\alpha \beta \alpha$ for some $\beta \in S$. Then $b=a \alpha=(b \beta) \alpha$ and $a=c \alpha=(a \beta) \alpha$ which implies $b \beta=a$ and $a \beta=c$. But, since $a<b$ and $c \& a$, this means $\beta$ is not order-preserving, a contradiction. Hence, $\alpha$ is not a regular element of $S$.

CASE 2. $X$ contains $\Pi_{2}$. Let $\alpha \in P(X)$ satisfy: $\operatorname{dom} \alpha=\{b, c\}$ and $b \alpha=b, c \alpha=a$. Then, $\alpha \in S$ since $\{b, c\}$ is isolated. Suppose $\alpha=\alpha \beta \alpha$ for some $\beta \in S$. Then $b=b \alpha=(b \beta) \alpha$ and $a=c \alpha=(a \beta) \alpha$ which implies $b \beta=b$ and $a \beta=c$. But, since $a<b$ and $c \neq b$, this means $\beta$ is not order-preserving, a contradiction. Hence, $\alpha$ is not a regular element of $S$. 
CASE 3. $X$ contains $\Pi_{3}$. The result follows in this case by recalling the notation at the start of Section 2 and applying Case 2 to $S(X, \leqslant$ opp $)$.

The next result now follows easily from the above Lemmas.

THEOREM 3.3. Let $X$ be a poset which is not a chain and suppose $S$ is one of the semigroups $O P(X), O I(X), O P\left(X, \aleph_{0}\right)$ or $O I\left(X, \aleph_{0}\right)$. Then $S$ is regular if and only if $X$ is isolated.

In [1, pp.27-33], Changphas provides various conditions under which $O T(X)$ is or is not regular when $X$ is not a chain. We summarise some of that work in the following three results without proof.

Theorem 3.4. Suppose $X$ is a poset. Then $O T(X)$ is not regular if $X$ contains a subposet of the form

$$
\{a, b, c, d:\{a, b\} \text { is isolated, and } d<c<a \text { and } d<c<b\}
$$

or

$$
\{a, b, c, d:\{a, b\} \text { and }\{b, c\} \text { are isolated, and } d<c<a \text { and } d<b\} .
$$

Theorem 3.5. Suppose $X$ is a poset and let $m(X)[M(X)]$ denote the set of all minimal [maximal] elements of $X$. Then $O T(X)$ is regular if $X=m(X) \cup M(X)$ and $x<y$ for all $x \in m(X)$ and $y \in M(X)$.

THEOREM 3.6. Suppose $X$ is a poset with a minimum element 0 and a maximum element 1. Then $O T(X)$ is regular if $\{x, y\}$ is isolated for all distinct $x, y \in$ $X \backslash\{0,1\}$.

\section{REFERENCES}

[1] T. Changphas, Regular order-preserving transformation semigroups, M.Sc. thesis (Chulalongkorn University, Thailand, 1998).

[2] D.F. Cowan, 'Partial cross-sections of symmetric inverse semigroups', Internat. J. Algebra Comput. 5 (1995), 259-287.

[3] C.C. Edwards and M. Anderson, 'Lattice properties of the symmetric weakly inverse semigroup on a totally ordered set', J. Austral. Math. Soc. Ser. A 31 (1981), 395-404.

[4] V.H. Fernandes, 'Semigroups of order-preserving mappings of a finite chain: a new class of divisors', Semigroup Forum 54 (1997), 230-236.

[5] G.U. Garba, 'Nilpotents in semigroups of partial one-to-one order-preserving mappings', Semigroup Forum 48 (1994), 37-49.

[6] G.U. Garba, 'Nilpotents in semigroups of partial order-preserving transformations', Proc. Edinburgh Math. Soc. 37 (1994), 361-377.

[7] G.U. Garba, 'On the idempotent ranks of certain semigroups of order-preserving transformations', Portugal. Math. 51 (1994), 185-204. 
[8] G.M.S. Gomes and J.M. Howie, 'On the ranks of certain semigroups of order-preserving transformations', Semigroup Forum 45 (1992), 272-282.

[9] P.M. Higgins, Techniques of semigroup theory (Oxford University Press, Oxford, 1992).

[10] P.M. Higgins, 'Idempotent depth in semigroups of order-preserving mappings', Proc. Roy. Soc. Edinburgh Sect. A 124 (1994), 1045-1058.

[11] J.M. Howie, 'Products of idempotents in certain semigroups of transformations', Proc. Edinburgh Math. Soc. 17 (1970), 223-236.

[12] J.M. Howie, 'Combinatorial and probabilistic results in transformation semigroups', in Words, Languages and Combinatorics, II (Kyoto, 1992) (World Sci. Publishing, River Edge, N.J., 1994), pp. 200-206.

[13] T.G. Lavers, 'The monoid of ordered partitions of a natural number', Semigroup Forum 53 (1996), 44-56.

[14] B.M. Schein, 'Products of idempotent order-preserving transformations of arbitrary chains', Semigroup Forum 11 (1975), 297-309.

[15] E.G. Shutov, 'On semigroups of almost identical transformations', (in Russian), Dokl. Akad. Nauk. SSSR 134 (1960), 292-295; English translation in Soviet Math Doklady, 1 (1961), 1080-1083.

Department of Mathematics

Chulalongkorn University

Bangkok 10330

Thailand
Department of Mathematics

Khon Kaen University

Khon Kaen 40002

Thailand 